\title{
Aspectos teórico-metodológicos sobre los procesos de reasentamiento poblacional e impactos sociales de la construcción de grandes represas hidroeléctricas
}

\author{
Alejandro O. Balazote
}

Unıversıdad de Buenos Aures Buenos Aires Argentuna abalazot@fibertel com ar

\section{Juan Carlos Radovich}

Unıversıdad de Buenos Arres Buenos Aires Argentına radovich@mall retina ar 
En este articulo nos proponemos analyzar algunas cuestiones relacionadas con los enfoques teorico metodologicos aplicados al estudio del impacto social de las grandes represas hidroelectricas Posteriormente nos dedicamos a desarrollar algunos aspectos destacados del aporte del Prof Sílvio Coelho dos Santos en este campo durante su dulatada carrera profesional

Palabras chaves hidroelectricas im pacto social reasentamientos teorias
In this article we propose to analyze certain questions related to the theoretical and methodological approaches of the study of social impacts of large scale hydroelectric dams Subsequently we expand upon some outstanding features of Prof Silvio Coelho dos Santos contributions to this field during his long professional career

Keywords hydroelectric dams social impact re localization theory 


\section{Introducción}

ב 1 Profesor Sílvio Coelho dos Santos fue uno de los investıgadores represas en América del Sur, conjuntamente con el Prof Leopoldo J Bartolome, quienes han liderado un grupo de antropólogos untegrado por investıgadores de Brasıl, Paraguay y Argentına, los cuales a través de encuentros academicos y publicaciones diversas han presentado los resultados de sus trabajos de investıgación Tambien han colaborado con su experiencia academica en la formación de recursos humanos especialızados, hecho que ha acrecentado la cantıdad de academicos dedicados a esta tematica Asımısmo, la partıcıpación de estos unvestıgadores en diversos encuentros ${ }^{3}$ posıbılıtó un provechoso intercambıo que se plasmó en distıntas publicaciones (Balazote, Catullo y Radovich 2001, Santos y Nacke 2003, Rets, Radovich y Balazote 2005, Verdum 2007) En todas estas publicaciones colectıvas Sílvıo partıcıpo con su valıoso aporte, producto de su vasta experiencia

A contınuacion desarrollaremos algunas cuestiones relacionadas con los enfoques teorico-metodológıcos aplıcados al estudıo de los impactos sociales causados por la construccion de hidroeléctricas y finalızaremos con el aporte realızado por el Prof Sílvıo en este campo

\section{Los procesos de reasentamiento de población}

Los reasentamientos forzosos de población orıgımados en la construcción de grandes proyectos, como es el caso de las megarepresas hidroeléctricas, ha sido uno de los tıpos de problemáticas mas estudıados por las Ciencias Socıales y en partıcular por la Antropologia Social Estos proyectos frecuentemente han afectado 
en muchos casos a poblaciones habitualmente estudiadas por los antropólogos (undígenas, campesınos, pobres urbanos), motıvo por el cual se han visto involucrados en los estudios de impacto social causados por las grandes obras desde la década de 1960 aproxımadamente Tal es ası que existen autores que han pasado a llamar de "antropología de las presas" a esta nueva y partıcular especialıdad (Barabas, 1992), más alla de las diferentes posicıones filosóficas, ideológıcas o teorico metodologicas sustentadas por los distıntos antropólogos involucrados en este tıpo de problemátıca

En cuanto a la relación que se establece entre antropologos y grandes obras, Rubeiro desarrolla un problema que considera como "una tensıón clasica de la Antropologia", y que, sin pretender solucionarla, no la desconoce Se trata de la utılidad del conocimiento y la práctica de los antropólogos unvolucrados en este tıpo de cuestıones (Ribeiro, 1990) Este autor considera que la problemática de las grandes obras y sus consecuencias sociales constituyen el nucleo temátıco fundamental en el cual se da, sea en mayor o menor grado, la transformacion del conocimiento antropológico en politicas publicas y en realıdades tangıbles Pero en esta misma característıca radica, según el autor, la gran dıfıcultad de una dıscıplına preponderantemente académıca, esto es, de qué manera el conocimiento antropologico puede influir en las prácticas de los actores sociales

En el caso de las grandes obras de infraestructura y sus efectos sociales, Ribeiro desarrolla una tupología sobre la base del grado de involucramiento y compromiso del antropólogo ante el proyecto Por un lado menciona la posición de "crítıca-sin adhesıón", en la cual el investigador se ubica como un "outsıder" y por otro la de la "criticacon adhesion", en donde la posicion del antropologo remite a la del "insider" (Rubeiro 1990 4-5)

Este tipo de proyectos, por otro lado responde a un modelo cuya lógıca, tal como la caracterızan Suárez, Franco y Cohen (1984), descansa sobre una sola variable la hidroeléctrica, y se muestran sumamente rígıdos a la hora de consıderar la uncorporación de otras dumensiones, a pesar de ser representadas frecuentemente como "presas de propósito múltıple' Evıdentemente, esta denomunación tıende a justıficar la 
gran inversión de capital que demandan estos proyectos y los costos sociales y ambientales que los mismos ocasıonan, ocultando la prioridad fundamental de producir hidroelectrıcıdad

Leopoldo Bartolomé, por su parte, y a raíz de su amplia experiencia en este tipo de problematicas, ha planteado toda una serie de consıderacıones en torno a los procesos de relocalızación (Bartolomé 1983, 19841985 a, b y c, 2000 y 2001) Este autor elabora sus propuestas a partur de sus estudıos realızados sobre reasentamentos poblacionales en el ámbito urbano, como en el caso de Yacyretá, hidroeléctrica bınacional emplazada entre Argentına y Paraguay sobre el río Parana, cuya construcción motıvó la necesıdad de reubicar un total de 40000 de personas

Al caracterizar los procesos de relocalızacion de poblacion, Leopoldo Bartolome plantea la necesıdad de distınguır entre los térmınos "( ) desplazamiento, la evicción de personas sun compensación adecuada o intento de planificar el proceso, y reasentamiento, donde existe por lo menos la intencion de planificar el proceso y mitıgar sus consecuencias negatıvas" (Bartolomé 2000 3-4) Asımısmo, señala al caracterizar estos procesos como consecuencias de "decisıones de desarrollo", que"( ) los desplazamientos ( ) se insertan claramente en campos politicos que involucran la operación de factores tales como el poder social y su distrıbución, ası como otros atınentes al diverso grado de agencia', (basándose en Gıddens para defunur este últımo concepto), como la real capacidad de un agente social para influenciar sus curcunstancias naturales y sociales, mas alla de su asignacion formal de poder" (Giddens 19845 )

Ribeiro por su parte, y en relación con su experıencia en el análısıs de la hidroeléctrica de Yacyretá, se diferencıa de aquellos autores que priorizaron en sus análısıs profundızar en los procesos de relocalızación y sus efectos sobre las poblaciones y comunıdades afectadas, concentrándose en enfatızar en su analısis las características de los "proyectos de desarrollo" que el denomina "Proyectos de Gran Escala" (PGE) (Rubeiro,1985) Destaca la partıcıpación de diferentes actores socıales y señala la dıstıncıón de diversos nıveles de intervención (ınternacionales, nacionales, regionales y locales ), siendo su intencion 
“( ) localızar y delımitar empíricamente las redes sociales que efectıvamente realızan las conexıones entre aquellos diferentes nuveles de poder ( )" (Rubeiro, 1991 17) Dicho concepto de PGE, se caracteriza por la presencia de tres rasgos distintivos "1) el aislamiento, 2) el gigantismo y 3) la temporariedad" (1985) De este modo, los PGE constituyen una intervención, nacional o binacional, con una marcada presencia de organısmos internacionales y que poseen, además, una clara intencionaludad geopolítıca Por otra parte, exıgen disponer de elevadas sumas de capital y la provisión de una gran cantidad de fuerza de trabajo El "gigantısmo" de estos emprendumientos requere el accionar de "( ) una corporación grande y poderosa" que maneje todo el proceso (Rıbeiro, 1985 34) Este autor también considera que el aislamiento de estos proyectos es relativo y está relacionado más bien a aspectos socioeconómicos (carencia de infraestructura, dificultades para contratar mano de obra caluficada, etc), más que geográfıcos (debıdo al usual aislamiento fisico de la regıon donde se construye la gran obra) (Ribeiro, 1985 34-35) Asımismo, el territorio donde se lleva a cabo el proyecto puede considerarse como una zona de "enclave"

Con respecto al tercer punto de este modelo la "temporariedad", el autor señala que los PGE constıtuyen sıstemas identıficables en el tıempo, poseeen un comienzo y una estımación aproxımada de finalızación La carga simbólıca de algunos puntos claves de este proceso, tales como la fecha de inauguración y su utılızación como acelerador del ritmo de obra, así como tambien el contenıdo polítıco de la ceremonıa de inauguración, no hacen más que confırmar el caracter temporal de esta modalıdad productıva

Aclara también, que la categoría de PGE, sólo se refiere a las caracterıstıcas de dimensión y planuficacıon Ahora bien, otro rasgo distuntıvo de estos proyectos, ademas de los mencionados más arriba, es el de constituir "totalidades discretas" ya "( ) que se trata de un conjunto organızado de relaciones con una lógıca partıcular, aunque inserto en un sistema mayor que es la fuente de sus principales características estructurales', y por otro, que componen totalıdades recurrentes", dado "( ) que hay sımılitudes entre los distintos PGE que justifican ubicarlos en una misma categoría" (Ribeiro, 1987 7) 
Otro aporte importante de este autor, luego de caracterizar a los PGE como una forma particular de produccion, es su analısis acerca de los componentes ideológıcos que estos proyectos poseen Señala que los PGE se legituman mediante el concepto de "Ideología de la redención", "( ) cuya matrız principal es la ıdeología del progreso, que muchas veces toma la forma del desarrollismo, es decir la suposicion de que los PGE son positivos porque desarrollarán una región, sumınistrando bienestar a toda su población Los elementos condensados en esta formulacion ideológica varían según la naturaleza de la obra, su importancia para la regıon o el país, y las partıcularıdades histórıcas y culturales del medıo en que se levantara" (Rıbeiro, 1987 Il)

En EEUU, Thayer Scudder y Elızabeth Colson realızaron los primeros trabajos antropológıcos sobre relocalızacıones poblacionales ocurndas en diversos paises y continentes Estos autores han elaborado un modelo de analisıs a traves del estudio de distıntos procesos de relocalizacion (Scudder 1975, 1981, Scudder y Colson 1978, 1982) Plantean una serie de hipotesis acerca de como puede esperarse que las comunidades, los grupos domesticos y los indıviduos respondan ante el reasentamiento forzoso y señalan algunas implicaciones que estas hıpótesıs producen sobre las polítıcas en la materıa Se trataría, segun los autores, de una serie lımitada de respuestas generalızables para los casos más varıados

Scudder y Colson (1982) distınguen dos tıpos diferentes de relocalızaciones las que sufren los refugiados y las "relocalızaciones para el desarrollo" Las prumeras incluyen a las victımas de guerras o de desastres naturales y se diferencian de las segundas debido a la imposıbılıdad de llevarlas a cabo mediante una adecuada planıficacion $\mathrm{Al}$ referirse a los reasentamientos poblacionales motıvados por las grandes represas, los incluyen entre las 'Relocalızaciones para el desarrollo", en la creencia de que las políticas relacionadas con los grandes emprendumuentos están destınadas a beneficıar a los afectados Evidentemente esta calıficacion resulta inadecuada, debido a que dicho concepto no explıcita con clarıdad cuáles son los sectores sociales que se benefician con el proyecto y quiénes resultan perjudicados con el supuesto "desarrollo" Debe tenerse en cuenta además que no exıste 
una "ley de desarrollo" homogenea, que produzca un impacto regular en todos los sectores involucrados Por ello resulta necesarıo entender las tensiones y contradicciones inherentes al impacto desigual que provocan los grandes proyectos, distinguiendo los efectos diferenciales que pueden ayudar a profundizar o exacerbar las contradicciones

A su vez este enfoque se ve permeado por la 1dea de "progreso" con la que estas grandes obras son sustentadas, sin tener en cuenta que, tal como afurma Leopoldo Bartolomé “( ） es demasiado frecuente que la relocalızación de la poblacion afectada sea concebida como un componente perfferico, como un costo adicional y de alguna manera '1legitumo'del proyecto global' (L Bartolome, 1983 7)

En la mayoria de estos grandes proyectos, en los cuales se enfatza la centrahdad de la obra de ingeniería, y cuyo princıpal objetıvo no es favorecer a los relocalızados, la reubicación de la población afectada, surge como una consecuencia de la obra respectiva, como un problema secundano a resolver dentro de la lógica general del proyecto Contrariamente a este enfoque, basado en cierto determinısmo tecnológıco, creemos tal como afurma L Bartolome que "( ) las relocalızaciones poblacionales constituyen una parte del todo complejo conformado por los componentes, durectos e indirectos, del Proyecto Global" (1983 2)

Por otra parte, el termino 'represados", acuñado por Scudder (1981), resulta de suma utılıdad para reflejar el drama social que los procesos de relocaluzacion compulsiva provocan A su vez, este autor elaboro el concepto de "Stress Multıdımensıonal de Relocalızacıón" (SMR) aplıcado al análısıs de los efectos del reasentamıento sobre la población afectada De este modo partiendo del supuesto de que toda relocalızación produce stress, analıza los dıstıntos componentes del mismo (fisıológıco, sociocultural y psicológıco), y los consecuentes efectos que se manıfiestan en cada uno de estos planos Este modelo psicosocial supone una permanente adaptabılıdad psicológıca y emocional de los represados quienes transitarían por una serie de etapas con una elevado nivel de stress para luego lograr el equilıbrio alterado, mediante la adpatación

De este modo, el SMR desde un punto de vista fisıológico incrementaría las tasas de morbilidad y de mortalıdad, antes, durante 
y despues de producıda la relocalızación Luego, el componente psicológıco se manıfestaría a través de una sene de sındromes, como el de "la pérdıda del hogar", entendiendo al hogar como la comunıdad en su conjunto y los síndromes de "ansiedad" ante un futuro incierto y el de "depresion" como efecto de los potenciales daños que se van percibiendo en el plano individual

Desde el componente sociocultural a su vez, el SMR es menos cuantıficable que los dos anterıores dado que esta asociado a los aspectos economicos, polítıcos y culturales del proceso de relocalızación Esta dımensıon se manıfıesta básıcamente modıfıcando la estructura de lıderazgo local, la red de relaciones socıales, las estrategıas adaptatıvas y al indıviduo afectado por la relocalızación (Scudder y Colson 1982) El deterıoro de las estrategıas de vida y la ruptura o crisıs de la estructura de liderazgo, señaladas por los autores expresan negatıvamente cıertas partıcularıdades de los procesos de relocalızación y, mas alla de su constatacion empírıca, nada especifican acerca de cuáles son las nuevas formas de reproducción desarrolladas por los afectados $n$ mencionan las condiciones, gestadas a raiz del proceso, que facilitan la emergencia de nuevos dirigentes, cuyas funciones y desarrollo variaran de acuerdo a su relación con el proyecto de relocalızacion imbricado en una coyuntura determunada

Otros autores que utılızan también el concepto de stress para el estudio de los reasentamentos poblacionales son Werner (1985) y Lumsden (1975) Lumsden aclara cuan diversos han sido los usos dados al termino stress y su trabajo se refiere a la descripcion del impacto producido por la gran represa Kainjı construida sobre el río Volta en Ghana

Sin embargo, podemos generalizar que todos los usos dados al concepto de stress en el tema que nos convoca, han sido tomados de las ciencias bıológıcas y aplicados a estudıos psıcologıcos, sociológıcos $Y$ antropológicos Todos los autores en los que se apoya Lumsden (Selye, Leighton, Smelser, y Lazarus) se basan en el paradıgma biologicista para elaborar su marco conceptual (Lumsden 1975 196) Según este autor "Las estrategıas se constıtuyen a partir de las regularıdades que un sistema evidencia en sus reacciones frente a los estimulos del medio con el cual interactua" (Lumsden 1975 195) La fuerte vinculacion de 
los térmınos estımulo-reacción y la noción de sıstema de regulandades constatan la influencia de cierto determinismo biologicista en los postulados desarrollados por Lumsden y otros autores, cuyo resultado consıste en la naturalızación de complejos procesos socioculturales

Asimismo el concepto de "stress psicosocial" elaborado por Werner, merece dedicarle algunas consideraciones critıcas Este autor pretende cuantıficar algunas de las diferentes formas que adoptaria el stress con el fin de realızal "comparaciones interculturales" que posibiliten poner en evidencia los "costos sociales ocultos" que siempre están presentes en los procesos de relocalızación (Werner 1985 161) Para ello aplica dos cuestionarios, uno compuesto de 20 items, tratando de delinear la presencia de "stress psicosomatıco" entre los individuos afectados y el segundo, basado en 13 preguntas, durigido a detectar el stress social" (Werner 1985 163)

Es necesario destacar que la mayona de los usos dados al concepto de stress, aplicado al estudıo de los procesos de relocalızación, se basan en modelos sistémicos que consideran a los grupos o comunidades afectadas como entıdades aisladas del todo social mayor, en general homogéneas y en constante equilibrio De este modo, un estímulo "externo" como la relocalizacion forzosa se introduce en dicho sistema u "organismo", provocando diversas reacciones sobre los distıntos elementos dcl sistema o sobre éste en su conjunto Estas reacciones se traduciran en respuestas adaptatıvas o maladaptatıvas Estas ultımas de repetirse contunuamente pueden provocar la destruccion del "sistema adaptatıvo complejo', pero este posee mecanısmos de defensa que posıbılıtan la recuperación del equilıbrıo perdıdo a través de la "autoregulacion" (Lumsden 1975 193)

Si bien esta ıdea de "sistema adaptatıvo complejo" está concebida como "sistema abierto ', sujeto a la "transacción" con el medıo o entorno, posibilitando el intercambıo "materıal", de "energía" y de "información", el conjunto social es visualızado como encapsulado, limitando o diluyendo la penetracion en estos sistemas de conjuntos sociales más amplıos, otorgando a los aspectos externos a los mismos, un rol meramente contungente Ası al priorizar en el análısıs los intercambıos que se producen mediante las transacciones reducidas a 
los ámbitos energétıco, materıal e informatıvo, se viabilıza el ocultamiento de otro tipo de relaciones, sobrevaluando a estos sistemas en si mismos e independizandolos de los procesos sociales que ocurren en la sociedad en su conjunto Se trata por lo tanto, de un modelo empirıcısta y ahıstorıco tendiente a una naturalızación unductıvista, que no contextualiza las particularidades ni toma en cuenta los aspectos estructurales condicionantes de todo proceso de relocalización

Scudder y Colson ademas, caracterizan a la relocalizacion como proceso, pero entendiendo que la contınuidad temporal se produce durante la relocalızación exclusivamente, reduciendo la idea de proceso a una serie de etapas secuenciales contenidas en el sistema afectado Las etapas o estadıos sugerıdos por estos autores son 1) reclutamiento, 2) transición, 3) desarrollo potencial, y 4) transferencia/incorporacion Las mismas comprenden desde la dıfusıón de las primeras notıcıas acerca de la gran obra y la relocalızación consecuente, hasta la adaptacion de los relocalızados en el nuevo asentamiento, una vez que se ha eliminado el impulso estresante dentro del sistema (1982)

L Bartolomé critıca esta perıodızacıón, en especial lo que refiere a la etapa 4) "transferencia/uncorporacion", en la que "( ) los reasentados se hacen cargo totalmente de los beneficios producidos por la gran obra y se incorporan plenamente como parte integral a la región en que están ubicados" Para este autor, "Por un lado son muy excepcionales los casos de agencias cuya intervencion activa se extienda por períodos tan prolongados, y menos aun aquellas vinculadas a o integrantes de, entes constructores de represas De plantearselo como modelo genenco, nos encontrarıamos con que en muy contados casos se cumplirían los requisitos postulados por Scudder para esta etapa, con lo que perdería gran parte de su utılıdad analítıca" (L Bartolomé 1983 24)

El mismo autor también critica el concepto de "equilibrio' vigente en el modelo de Scudder ya que se trataría de "( ) un proceso dinamico y cuyas 'fronteras no estan de nunguna manera limitadas a las trayectorias de los relocalızados', ( ) y sugiere "( ) incorporar en forma más explícita al modelo las variables 'interactıvas', es decur, aquellas que se orıgınan en la unteracción entre la o las comunıdades relocalızadas y sus contextos" (L Bartolomé 1983 25) 
Otra critica al modelo de Scudder proviene de Partrıdge, quien afırma que el mismo ha servido muy poco para las necesıdades operacionales de todo proceso de relocalización (1989)

Según Barabas, de acuerdo con estudios realızados en torno al proyecto Guavio en Colombia, en el cual se aplico el modelo de Scudder y Colson, se pudo percıbır, varıos años después de producıdo el reasentamento, “ ( ) [que] los niveles de desesperanza y ansiedad de los afectados, así como los de disgregación familuar y social eran altos, y que no existian claras tendencias a la recreación de pautas de relación social" (Barabas 1992 310), por lo que el incremento de las capacidades de respuestas colectivas e indıviduales no constituyen respuestas recurrentes en todo proceso de relocalızacion De acuerdo con esta autora, "El énfasis en la capacidad de respuesta de los relocalizados, en tanto actores actıvos y creatıvos del proceso de cambıo llevó tal vez a visıones demasıado optımıstas y confiadas sobre la 'adaptabilidad hacia el equilıbrıo' de los grupos dislocados" (Barabas 1992 310)

Respecto a la afurmación de Scudder y Colson (1982) relacionada con el "debulitamiento de los liderazgos locales" ante el proceso de impacto, hemos podido comprobar, a partur de nuestras unvestigaciones llevadas a cabo entre 1987 y 1992 en la agrupacion mapuche Plquinyeu del Limay, afectada por la construcción de la hidroelectrica Pıedra del Aguila en la patagonıa argentina, el fortalecumiento de los liderazgos locales como estrategia de respuesta colectiva ante la inminencia del reasentamiento forzoso (Balazote y Radovich 1993)

De Wet por su parte tambien critıca el modelo descripto, al señalar que "( ) opera en un elevado nivel de generalidad el cual limita su habilidad para tenerlo en cuenta para diferentes tupos de respuestas a la relocalızación en dıstıntos contextos, partıcularmente después de que las comunıdades han ingresado al estadıo de desarrollo potencıal' (1988 182) Incluso el propio Scudder sugiere que su modelo opera con mayor utılıdad en las fases de reclutamiento y de transıción del proceso de relocalización (De Wet 1988 182)

De Wet en cambio, propone un analısıs medıoambiental para aplicar a los procesos de reasentamiento de comunidades basado en los comportamientos de los afectados, perspectıva que, según este au- 
tor Scudder y Colson no toman (1982) Segun De Wet, el exito de su abordaje ambientalısta radicaría en que posibilitaría entender por qué algunas experiencias de reasentamiento han sido mas estresantes que otras La respuesta, de acuerdo con este autor estaria relacionada con las modificaciones causadas al ecosistema Por lo tanto cuanto mayor sea el cambıo producıdo, mas intenso será el stress correspondiente (1988)

Otro aspecto no tenido en cuenta por el modelo de Scudder y Colson, entre otros, es que "( ) no se presta especial atencion a la alterıdad cultural" (Miguel Bartolomé 1992 26) Segun este autor, "Entre la explicaciones posibles de estas omisiones, deberiamos tal vez mencionar el énfasıs 'campesınısta y economicısta, atrıbuible a las cıencıas socıales en las últımas dos décadas, lo que las llevo a mınusvalorar la cuestıón etnıca, adjudıcándole el carácter de una varıable secundarıa" (M Bartolome 1992 26)

Michael Cernea, a su vez, realıza en 1988 una reflexıón critıca en torno a las formas erroneas adoptadas por el Banco Mundıal (BIRF) en diversos procesos de reasentamiento derıvados de grandes proyectos funanciados por esa instıtución Cernea planteó el problema de la "patología del desarrollo" (1997), consistente en la intensificacion de un proceso de empobrecimiento que se manifiesta en diversas dimensiones y que padecen los afectados por los grandes emprendimientos 'Indudablemente, los reasentamientos involuntarıos constituyen un dominio en el cual el clamor por la justicia social y la distribucion equitativa de los beneficios del desarrollo resuenan muy bajo" (Cernea 1997 1570) Con esta afurmacion el autor pone el acento en una de las cuestıones mas recurrentes en este tıpo de proceso, o sea, la "injusticia social" y la desigualdad inherentes al "desarrollo"

Debido a lo expuesto y luego de mas de 15 años de investıgaciones, Ceinea elabora un modelo explicativo de los procesos de relocalizacion basado en los conceptos de "rıesgo" y "reconstrucción Afırma enfátıcamente que ante estos complejos fenomenos " ( ) el empobrecimiento (de los relocalızados), no constıtuye una fatalıdad y no debe ser tolerado con pasıva resıgnación ( ) El desplazamiento forzoso es una disrupción causada socialmente y no un desastre natural y sus efectos perversos deben y pueden ser contrabalanceados (Cernea 
1997 1570) Por otra parte critıca también la supuesta "inevitabilıdad" de los reasentamientos y sugiere la alternatıva de enfrentar los riesgos cuando afirma que "( ) no todos los casos de desplazamientos propuestos por los planificadores son inevitables o justificados ( ), (y) los reasentamientos conducidos con un instrumento de equidad pueden contrarrestar el empobrecumiento, generando beneficios, tanto para la economía regional como para la local (Cernea 1997 1570) De esta manera, Cernea plantea un "modelo de reconstruccion" que tıene en cuenta el concepto de "rıesgo" basado en la definıción de Giddens, quien lo entiende como "( ) la posibilıdad de que cierto curso de acción podrá disparar futuros efectos pernıcıosos, pérdıdas y destrucción" (1993)

El modelo propuesto se trata tanto de una herramenta para generar y organızar el conocimiento como ası tambien de una guia para la acción en la gestión de los proyectos Segun el autor, cumple con un rol cognitivo y operacional que se puede complementar con otros modelos y aplicar tambien a otros casos de desplazamientos forzosos, tales como los refugiados por guerras y los reubicados debido a desastres naturales (Cernea 1997 1571) A su vez, el modelo consta de cuatro funciones 1) dıagnóstıca, que es a la vez explıcatıva y cognitıva, 2) predıctıva (útıl para la planıficación), 3) resolutıva de problemas (durante el proceso de relocalızación), y 4) investıgatıva, para la formulacion de hıpotesıs y elaboración de teorias (Cernea 1997 1571)

Ahora bien, la conceptualizacion clave de este modelo es la de "riesgos por empobrecimiento", los cuales pueden ser analızados a través de un dıagnostıco Los riesgos descriptos son falta de tıerra, trabajo y vivienda, marginalızacıón, incremento de morbılıdad y mortalıdad, insegurıdad alımentarıa, ımposibılıdad de acceso a la propiedad comunitaria y desarticulacion social (Cernea 1997 1572) Segun este autor, los rasgos detallados más arriba se constituyen en inminentes o amenazantes, previo al traslado de los afectados aunque tambien se haría extensiva su influencia en los niveles local y regional El autor realiza una comparación evaluatıva de distıntos procesos de reasentamiento llevados a cabo en diferentes lugares, aphcando este modelo basado en los riesgos del empobrecumiento de los desplazados 
de acuerdo a como fueron tomadas en cuenta las dimensiones descriptas en el parrafo anterior

Becker (1997), quien ha focalizado a los procesos de relocalizacion desde la perspectiva de la gestion y el asesoramiento de los impactos sociales relacionados, considera que los reasentamientos poblacionales constituyen procesos de transicion que llevan aproximadamente 15 años, desde que se difunden los primeros rumores acerca de un potencial traslado, hasta que los relocalizados se adaptan a la nueva situacion Asımismo, afirma que el asesoramiento en este tipo de impacto constituye un proceso largo, complejo y costoso Para este autor, frecuentemente el tiempo destınado para la planıficación y ejecución del proyecto suele resultar escaso y el inicio de la planificaicon generalmente se concreta en una etapa demasiado avanzada, cuando los conflictos y ciertos efectos iniciales ya se han instalado Por otra parte, considera que los costos de la planificación de la relocalızacion en térmınos financieros, equipamiento y personal tienden a duplicar las estımacıones presupuestarıas prevıas Señala tambıen que la ınadecuada planificacion financiera de estos proyectos se relaciona con el hecho que muchas veces el organismo responsable no reconoce nı calcula estrictamente todos los costos que insume el reasentamiento

En otro eje tematico la participación de los afectados es una de las cuestionts cruciales inherentes a todo proceso de reasentamiento poblacional

En este sentıdo, la propuesta de Partridge, al analızar un tema tan crítico y conflictivo en todo proceso de relocalización como es la partıcipación de los afectados enfatıza que los dos problemas mas apremiantes que deben solucionarse al programarse proyectos de relocalızacion son a) descubrur la organizacion social endogena de la comunidad amenazada por el desplazamiento" y b) "establecer un programa de partıcipacion de la comunidad en el proceso de reasentamiento que se adecue a esa organızación social endogena" (Partrıdge 1985 51) Luego de defunir a la comunidad humana en terminos de "regularidades de interaccion" y "comportamiento cultural" en un "contexto ambiental", se orienta a la caracterizacion de los "grupos corporatıvos", siguiendo la elaboracion de Smith, quien destaca la "( ) presunta perpetuidad, circunscripción, afılıacion restringıda identı 
dad, autonomía, organizacion, procedimientos y asuntos comunes" de dichos grupos (Partrıdge 1985 54) Posteriormente al centrarse en el problema del reasentamiento poblacional, propone como aspecto esencial incluir a los grupos corporativos endogenos en las distuntas instancias del proyecto de relocalización, aclarando que no basta la mera conciencia de la necesidad de partıcipacion, sino que es necesarıo elaborar una metodología coherente, involucrando a todos los afectados y aclarando que no se trata de invitar a la comunidad a participar, sino en como lograrlo Pero el meollo de la propuesta de Partridge se basa en conocer la organızación de dichos grupos para diseñar y organızar adecuadamente la competencia entre grupos corporatıvos endógenos y exógenos, con el objetıvo funal de evitar o munumızar la resıstencia y el conflicto que los grupos corporativos pueden instrumentar ante la relocalızación compulsıva Muchas veces, los errores que se cometen a la hora de otorgar partıcıpacion a los relocalızados o represados", tal como los denominan Scudder y Colson (1982), se deben a que “( ) se intenta operar en la dimension política, pero se parte del equívoco de considerar que la naturaleza de la accion politica en la sociedad nativa es similar a la del estado que la incluye' (M Bartolome 1992 23)

Otros autores señalan la importancia de la dıfusıón de la información como una premisa indıspensable para el logro de la partıcı pacion de los afectados "Resulta normal y esperable la existencia de resistencia y aún de hostılıdad inıcial hacia la idea de la relocalızación unvoluntarıa La naturaleza de las respuestas de los relocalızados y de la población anfıtriona dependerá, en gran medıda del establecumıento de buenos mecanismos de comunicacion y de la realızacion en forma regular de consultas con los grupos involucrados y con sus organizaciones (asociaciones locales, organizaciones no gubernamentales, etc), ası del alıento que se brınde a la partıcıpación de éstos en la busqueda y definicion de soluciones para los complejos problemas que emergen en la planıfıcación y ejecución de relocalızaciones Aun mas, la carencıa de ınformación precısa puede exacerbar las malınterpretaciones y fortalecer la resistencia al traslado (C€rnea 1989 20)

Por otra parte, la comunicacion por si musma no garantıza la participacion de los grupos afectados si no es acompañada por la in 
corporacion de los mismos en los ambitos donde realmente se toman las decisiones $\mathrm{Al}$ respecto, el mismo Banco Mundıal recomienda explicitamente a las agencias encargadas de relocalızaciones "Superar la tendencia burocratica a retener dentro de su esfera de competencia todos los aspectos de los procesos de toma de decisiones y de las funciones administratıvas ( )" (Cernea 1989 36)

Asimismo, es necesario aclarar que la comunicacion "per se" no dismınuye el nıvel de conflicto nı soluciona los problemas de los afectados Por otra parte, la participación debe ser instrumentada en la organızación comunitarıa a traves de sus instituciones de manera tal que esta lleve, con mayores posibilıdades, las negociaciones para obtener mejores condicıones para su relocalızación Ademas, cuando los afectados poseen un mayor grado de libertad para negociar su fu turo, el umpacto del traslado suele resultar menos perjudicıal

Evidentemente este aspecto debe ser entendıdo como un campo de actıvidad politıca donde se interrelacionan diversos intereses en niveles distıntos de actuación (Catullo 1996) Esta autora analızo y decribıo con profundidad este aspecto al estudiar los efectos sociales causados por la represa argentino-uruguaya de Salto Grande sobre el rıo Uruguay (Catullo 2001 y 2006, Catullo y Patt 2001)

Otro aspecto importante a tener en cuenta en este tipo de situaciones es la necesıdad de distınguir entre relocalızaciones rurales y urbanas Las diferencias entre ambos tipos de procesos se encuentran en las distuntas "estructuras de recursos" y en las variadas estrategias de reproduccion que en relacion a dichas estructuras desarrollan los grupos afectados Asımismo y siguiendo a L Bartolomé, consideramos que los aspectos laborales/productıvos en el diseño de programas de reasentamiento forzoso deben ocupar un papel predominante, ya sean llevados a cabo tanto en ámbitos rurales como urbanos (L Bartolomé 1984 b)

El Banco Mundial (BIRF) por su parte, responsable de la financıación de muchos proyectos de construcción de hidroeléctrıcas que ocasıonaron diversos impactos sociales negativos, tales como reasentamıentos forzosos de poblaciones indıgenas, asumıó en el año $1982^{4}$ una nueva orıentacion politica ante los graves perjuicios que sufrian las poblaciones afectadas Si bien el BIRF no impugnaba estructuralmente 
al modelo de aprovechamıento hidroenergétıco implementado hasta entonces, se vieron enfrentados a la necesidad de minimizar o atenuar los elevados costos sociales motıvados por esas grandes obras, en especial debido a las crecientes críticas y a los movimientos de oposicion que dichas grandes obras provocaron Hasta ese momento, las crítıcas se habían intensıficado sobre este organısmo unternacional debido a una visıón de corto plazo, que obviaba las condıcıones locales y que no consideraba las implicaciones a largo plazo para los grupos humanos y la biodiversidad De acuerdo con las critıcas, muchos proyectos de represas en el Tercer Mundo fueron claros ejemplos de "imperialısmo ecologico' , donde la explotacion de la gente y de los recursos naturales fue presentada como factores de "desarrollo'

En 1994 la 'Declaración de Manıbelı' 5 solıcito al Banco Mundial una moratoria a la financiacion de grandes represas en todo el mundo Asımısmo, se estıma que alrededor de 10000000 de personas fueron forzadas a reasentarse debido a proyectos de grandes represas financiadas por el Banco Mundıal Es entonces, que a partır de este tipo de críticas, el BIRF recurrio a la utılizacion de marcos conceptuales de las ciencias sociales para la implementación y evaluacion de los proyectos con el fin de evitar graves conflictos sociales y politıcos que se estaban produciendo en distıntos lugares del planeta Según Barabas, a instancias de estos nuevos lineamıentos asumidos por el BIRF ( ) los estados nacionales latunoamericanos, a traves de las instituciones y entes encargados de la realizacion de proyectos, han comenzado a cambiar sus propıas politıcas y práctıcas respecto a la relocalización de poblaciones afectadas por presas y a la proteccion del medıo ambiente asociado" (1992 307)

Estos nuevos lineamientos fueron adoptados ante la creciente preocupacion que generaban los proyectos asistidos financieramente por el Banco Mundial, con el objetivo general de fomentar el "desarrollo" de areas marginales en zonas rurales, pero que asımısmo afectaban las formas de vida de comunıdades indígenas de dichas areas Un documento del BIRF defınía como "pueblos tribales", a aquellos que ' ( ) contınúan viviendo $\mathrm{en}$ la perıferıa de la sociedad nacıonal domınante ( )' En este documento se sugıeren una serıe de medıdas orıentadas 
a salvaguardar la integridad y el bienestar de estas poblaciones a través del acuerdo con las mismas Plantea asımismo la necesidad de evitar llevar a cabo las medidas que tiendan a perpetuar el aislamiento o a promover una acelerada 'integracion/aculturación" forzada de esas poblaciones Teniendo en cuenta lo mencionado anterıormente, señala que las politicas del BIRF en relacion con las "poblaciones tribales", deben aplicarse en consonancia con los objetıvos y guías generales del Banco sobre todo en lo concerniente a las polítıcas sobre reasentamientos involuntarios de poblaciones, afectadas por proyectos asistidos por el Banco Mundial (Escudero 1988)

Las politıcas del BIRF defınıan como "poblaciones tribales", a los grupos étnicos con 'sistemas economicos estables" y de bajo sustento energétıco (Escudero 1988 11) Incluye como ejemplos a los cazadores/ recolectores agricultores semipermanentes, pastores y pescadores que posean en diversos grados las siguientes caracterıstıcas “a) geograficamente aislados o semiaislados, b) aculturados o solo parcialmente aculturados en las normas de la sociedad dominante, c) produccion no monetarızada o parcialmente monetarizada, mayormente destunada a la subsistencia e independiente de los sistemas economicos nacionales, d) etnicamente diferenciados de la sociedad nacional, e) lletrados y sin lenguaje escrito, f) distincion linguistica con la sociedad global, g) marcada identuficacion con un territorio particular $h$ ) estulo de vida economıco dependiente en gran medıda del medıo ambiente, 1) lidera zgos indígenas con escasa o nula representacion nacional y con escasos derechos como indıviduos o como colectıvidad, y j) habıendo perdıdo la tenencia de sus tierras tradıcionales las cuales en su gran mayorıa no son aceptadas por la sociedad nacional, incluso en las cortes y con debules capacidades de imponerse contra intrusos, uncluso cuando las áreas tribales han sıdo delımitadas" (Escudero 1988 11)

Evidentemente esta caracterización posee una impronta culturalısta muy acentuada, con componentes de dudoso valor a la hora de definir a un pueblo como tribal o "indigena" Esta vision internalista no escapa del frecuentemente citado 'mito bipolar' basado en la dicotomia desarrollista "tradicional/moderno" Incluso el uso del concepto tubal, jamas definıdo de manera adecuada, constıtuye un

\section{ILHA}


arcaısmo de escaso valor metodológıco, pero que coadyuva a cosificar y naturalizar los pueblos orıgınarıos Sin embargo las propuestas generales emanadas de las directivas del BIRF, a partır de los comienzos de la década de 1980 y posterıormente en 1991 (Uqullas y Davis 1996) con la aprobacion de nuevas normatıvas en lo que atañe a poblaciones indigenas afectadas por la construcción de grandes obras, constıtuye un avance y un atenuante para aquellos gobiernos en el denominado Tercer Mundo, los cuales han tenido una escasa sensibilıdad ante los efectos negativos producidos por los megaemprendumuentos sobre estos pucblos Es ası como la reposición equivalente de tıerras adicionales en cantıdad y calıdad semejante a las afectadas, es una de las directıvas principales sugerıdas por el BlRF

Posterıormente el Banco Mundıal elaboró una serıe de recomendaciones encamınadas a ajustar los procedımientos de evaluacion y monitoreo de los proyectos financiados que implican reasentamientos poblacionales Entre estas durectivas, el BIRF plantea la necesidad de cubrir toda la gama de impactos potenciales de un proyecto, el diseño de un adecuado programa de reasentamiento que contemple los aspectos legales, unstıtucionales, compensaciones por perdıdas, proteccıón del medıo ambiente y partıcıpacıon de los afectados en todos los aspectos del programa de reasentamiento (The World Bank 2001)

Sobre la base de estas recomendaciones según Barabas y Bartolome “( ) se ha logrado que en los últumos años algunos parses, como Méxıco y Brasıl, hayan comenzado a modifıcar sus procedımıentos de relocalizacion (1992 9) Asimismo, estos autores mencionan que "( ) la búsqueda de una eficacia operativa con frecuencia ayuda a mitigar situaciones injustas, que no son necesariamente generadas por voluntad sino por ineficiencia institucional" (Barabas y Bartolomé 1992 9)

Sin embargo, en otras ocasıones, la propıa polıtıca del Banco en materıa de funanciación de grandes obras está marcadamente sesgada a apoyar a los sectores hegemonicos comprometidos con la realızación del proyecto ${ }^{6}$ Ello nos lleva a consıderar las varıables connotaciones politıco/ınstıtucionales que poseen estos proyectos en el marco de polítıcas globales que no deben escapar del análısıs a la hora de evaluar los mismos 
Por su parte el Banco Interamericano de Desarrollo (BID), organısmo internacional cuya presencia en la financiacion de este tipo de proyectos ha sido muy frecuente, también promueve una nueva optica a la hora de encarar los reasentamientos poblacionales como consecuencia de la construcción de mega-represas Ası es que a partır de 1984, la posicion del BID sobre el reasentamiento involuntario de poblaciones comenzó a tomar nuevas formas dado que ese año los departamentos de Analisıs de Proyectos y Operaciones prepararon una serie de aspectos a ser tenıdos en cuenta durante el desarrollo de los proyectos que provocan relocalizaciones forzosas Posteriormente, en 1990 el Comite del Medıo Ambiente del Banco, para facllitar una revısıón más sıstematıca de los temas de reasentamıento, incluyo este aspecto en su campo de accion (BID 1996) Los nuevos lineamientos introducidos tenían como objetıvos principales, a) mitıgar los efectos negativos del traslado forzoso de personas y comunidades y b) prestar asistencia a las poblaciones afectadas para que establezcan una socie dad y una economia sostenibles" (BID 1996 1)

Otro instrumento importante a nivel internacional fue la aprobacıón del Convenı 169 de la Organıacion Internacional del Trabajo (O I T ), el cual constıtuyo una modificación del Convenıo 107 sancionado en 1957 por dicho or ganısmo sobre "la proteccion de los pueblos tribales y semitribales", modificando estas conceptualızaciones de fines de la decada de 1950 que, basadas en premisas culturalıstas e integracionıstas, pretendía “( ) facılıtar una acción para asegurar la protección de las comunıdades en cuestıón su integracion progresiva a las comunidades nacionales respectıvas y el mejoramiento de sus condicıones de vida y trabajo" (Plant 1988 7) El Convenıo 169, en cambıo aprobado en Ginebra en $1989^{7}$ cambia de rumbo en lo que respecta a la aceptación del derecho de los pueblos indígenas a contınuar con su modalıdad cultural y productıva En lo atunente a los grandes proyectos se establece la necesidad de implementar mecanısmos y procedımıentos de consulta con el fin de proteger territorios y recursos naturales Tambien se plantea que las grandes obras deben construurse siempre y cuando no exısta otra alternatıva menos intrusiva y perjudicial hacia los pueblos indígenas involucrados (Convenı 169, OIT) Un aspecto 
interesante de este cambio, desde un punto de vista conceptual, consiste en el reconocimiento de los indigenas como "pueblos" y su derecho a la "autonomia' y la "autodeterminación", aunque estas nociones fueron atenuadas en la declaracion final, al connotarlas con cierto grado de ambiguedad Asimismo la introduccion de conceptos como "autodesarrollo" y ' etnodesarrollo" undigena en consonancia con los reclamos indigenas a nivel internacional constituye otro logro de este instrumento a la hora de instrumentar reclamos relacionados con la implementacion de proyectos El Convenio establece que los pueblos indigenas no deben ser trasladados de las tıerras que ocupan A su vez, cuando excepcionalmente la reubicacion se considere necesaria, debera efectuarse con el consentimiento, consulta y participacion de los afectados Asımismo, consıgna que siempre que sea posıble, los pueblos indígenas deberán tener el derecho de retornar a sus territorios en cuanto dejaren de existir las causas que motivaron el reasentamiento (Art 16\%, Convenı 169, OIT)

\section{La producción académica de Sílvio Coelho dos Santos en relación con el impacto social de los grandes emprendimientos hidroeléctricos}

La produccion de este autor sobre esta tematica es muy abundante Nosotros solamente nos referiremos a algunos aspectos claves de su producción que han enriquecido la discusion entre los especialıstas

En un trabajo de 1983, Silvio Coelho dos Santos ya nos alertaba acerca del contraste que planteaba el gigantısmo de las grandes represas y la "( ) realidade de um país (Brasil) economicamente dependente e perıferıco" (Santos 1983 1) Asımısmo señalaba que en realıdad la politica energetica de su pais se dırıgia a satısfacer los intereses de los países avanzados antes que "( ) as efetıvas necessıdades da sociedade nacional (Santos 1983 1)

Consecuentemente, caracterizaron a los proyectos del sector eléctrico como resultantes "( ) de fato de inıcıatıvas complexas e multıdimensionais, compreendendo aspectos econômıcos, polıtıcos, socio-culturais tecnicos e ecologicos relacionados em um intrincado jogo de mútuas interações e condıcionamentos ( ) dependem tambem ( ) pelas suas dimensões, de grandes movimentos de capital e 
mão de obra Em consequência produzem profundas alterações em diferentes esferas que extrapolam seus aspectos meramente econômicos e técnicos (Santos y Reis 1993 2)

Por otra parte y en relacion con este tema, el Prof Silvio siempre mostró una gran preocupacion por la situacion particular que vivían los más perjudicados por estos emprendımientos, generalmente las poblaciones rurales e indigenas (Aspelın y Santos 1981), las cuales, segun este autor, generalmente no tuvieron un tratamiento diferenciado como se reconoce actualmente La construccion de las hıdroeléctrıcas de Tucuruı Balbına e Itaipu, durante la década de 1970, afecto seriamente territorıos indígenas, provocando diversos impactos sociales, tales como procesos de reasentamiento compul sivos con el consecuente deterioro de las condiciones de existencia de los afectados (Santos y Nacke 2003 a 11 )

En el sur de Brasıl, Sílvio señalaba específıca y minucıosamente las areas indigenas y los diversos pueblos (Guaranı y Kaingang) afectados por la construcción de hidroelectricas en los estados de Parana, Santa Catarina y Rio Grande do Sul (Santos 1983) Lo mismo efectuaba Helm para el area del no Tibagı, donde la represa Maua iba a producır severos impactos sobre territorio kaingang (Helm et al 1998, Helm 1999,2001 a y b)

Sin embargo, lo más destacado de dicho trabajo lo constıtuye el acapite dedıcado a Os Indıos e o Directo", denunciando el "régımen de tutela establecido por el Codigo Civil (Art $6^{\circ}$ inc III) en vigencia en esa epoca y aplicado acrítıcamente por la Fundação Nacional do Índıo (FUNAI) Asımısmo, Sílvıo señalo en este artıculo y en otros, la necesidad de tener especial cautela cuando se trata de territorios indıgenas dada la avide $\iota$ siempre existente y relacionada con intereses privados, señalando que los territorıos indígenas "( ) pertenecen a las sociedades indigenas, siendo inalıenables y no pueden ser utılızadas para la contınua expansion de la sociedad nacional (Santos 1983 21) Por otra parte, complementariamente indicaba la necesidad de llevar a cabo indemnizaciones justas y dignas", enfatizando que "Deve-lhes compensar terra por terra em lugares ıguais, benfeitoria por benfeitorı e bens naturaıs e custos socıaıs por indenızação mo- 
netarıa, reconhecendo aunda o dıreıto à partıcıpação permanente nos resultados financieros do projeto na forma de ações ou de beneficios outros destinados a compensar as perdas causadas Essas recompensas devem chegar dıretamente ao grupo e não ao orgão tutor e debe ser o grupo quem decide como usa-los" (Santos 198321 y 1996 b) Como puede apreciarse, en este párrafo se encuentran señalados los factores claves relacionados con la posibulidad de llevar a cabo una exitosa politica de compensacion, al tratarse de pueblos originarios y que finalmente durante los últımos años fueron adoptados en diversos paises de nuestra region latınoamericana

Sin embargo, y a pesar de dicho reconocimiento, los errores cometıdos en el pasado no dejan de impactar en el presente, tal como acontece con los pueblos guaraníes de Ocoí $\mathrm{Mbya}^{8}$, Ñandeva y Kaoiwá, afectados desde mediados de la decada de 1970 por la represa bınacıonal de Itaıpú, quienes sıguen padeciendo los efectos negatıvos de dicho emprendımiento tal como lo señalan Santos y Nacke, al comprobar el deterioro de las condiciones de vida y la falta de asistencia por parte de las agencias estatales (Santos y Nacke 2003 b)

La nueva problemática que comenzó a plantearse durante la década de 1990, con los procesos de privatızación de las hidroeléctricas, instalando un nuevo escenarıo en la arena polıtıca, fue analızada crítıcamente poı Sílvıo, cuando señalaba que 'A realıdade do processo de prıvatızação do setor elétrıco no cenarıo neolıberal aparece, assım por inteuro As noções de direıto dıfuso, de efeito global e externalıdade, ( ) não estão sendo consıderadas ( ) Tudo indıca que no cenárıo de privatızação ( ) havera pouco espaço para discutır os direitos das populações afetadas" (Santos y Henriques 2001 67) En este sentido, resulto sumamente pertinente plantear la utılızación del concepto de "efecto global" y el reconocimiento de "derechos difusos" por parte de los organismos responsables de la construccion de giandes obras hidroelectricas durante la difícıl coyuntura vivida durante la aplicacion de las polítıcas neolıberales

Finalmente, deseamos concluir este trabajo con una cita que ilustra claramente el pensamiento de Súvio y su involucramiento con la problemátıca estudiada a lo largo de treınta años de investıgaciones 
Aspectos teorico metodologicos sobre los procesos de reasentamiento poblacional e impactos sociales de la construccion de grandes represas hidroelectricas

"Não basta se pensar os projetos hidroeletricos como de interesse da melhorıa da qualıdade de vıda da maıorıa da população do país, de um estado ou de uma região E precıso assegurar aqueles que são prejudicados por taıs projetos, devido à desaproprıação de suas propriedades, por seu reassentamento forçado, por perda de empregos e de relações de vizınhança, entre outros efeıtos negatıvos, que tenham efetıva oportunıdade de reconstıtuırem suas condıções de vida, em termos socio-culturais e econômicos ( ) Necessarı, pois se ter clareza que os projetos hidroeletricos que tanto têm permitido a expansão econômica da sociedade como um todo, também têm faces sombrias que necessitam permanentemente atenção e monitoramento (Santos 2007 52-53)

\section{Notas}

1 Los mismos se vienen produciendo desde 1995 cuando se organizo en el marco de las reunıones de la Asociacıon Brasıleña de Antropologıa del sur del pass (ABA Sul) el primer encuentro de antropologos del Mercosur Posteriormente en las diversas Reuniones de Antropologos del Mercosur (RAM) llevadas a cabo en Pirıapolis 1997 Posadas 1999 Curitiba 2001 Florianopolıs 2003 Montevideo 2005 y Porto Alegre 2007 se fue afianzando el espacio dedicado al estudio de la problematica social de las hidroelectricas en este marco academico plurunacional

- Precisamente un ano despues que Robert McNamara dejara de presıdurlo luego de un periodo de 13 años cuando las nuevas autorıdades asumen las critıcas dırıgidas al economicismo del Banco Mundial y aceptan la reivindicacion de otros aspectos del desarrollo humano (educacion salud derechos humanos etc) (The World Bank 2001)

3 Dicha declaracion fue firmada por diversas redes grupos y coaliciones de 44 paises con un total de 2154 ONG s (McCully 1994)

4 Tal es el caso de la presa Pangue sobre el Alto B1o Bio en Chile donde la International Finance Corporation integrante del grupo Banco Mundial aporto sus recursos a solicitud de los interesados ( ) con el fin de aumentar la credıbılıdad de la empresa chilena en el mercado internacional de capitales (dado) que esta fuente de financiamiento contribuiria a legitimar un proyecto problematico en cuanto a sus impactos ecologicos e impugnado con mucho fervor en el pais y en el extranjero (Robinson 1992 86)

5 La Argentına tardo mas de 10 años en aprobar y ratıficar el Convenı No 169 de la OIT Asimismo todavia queda pendiente su aprobacion en algunos paises de America Latina

6 Para conocer el proceso de impacto sufrido por los Mbya como consecuencia de la represa binacional de Yacyreta ver Rehnfeldt (2003) 


\section{Bibliografía}

ARACH Omar Fntre rios si/entre represas no la paiticipacion ambienta lista en la oposicion a la represa de Parana Medıo (Entre Rios 1996/97) In BALAZOTE, A CATULLO M RADOVICH J C (Org) Antropologia y grandes proyectos en el Mercosur La Plata Minerva 2001

ASPELIN Paul SANTOS Silvio C dos Indian areas threatened by hydroelectric projects in Brastl Copenhagen IWGIA 1981 (Document 44)

BALAZOTE Alejandro RADOVICH Juan Carlos Gran obra e impacto social en Pilquiniyeu Buenos Aures CEAL 1993

BALAZOTE Alejandro CATULLO Mana Rosa RADOVICH Juan Carlos (Org) Antropologia y grandes proyectos en el Mercosur La Plata Minerva 2001

BARABAS Alicia Chinantec messianism the mediator of the Divine In SEVILLA CASAS E (Ed) Western expansions and indigenous peoples Nether lands Mouton Publıshers 1977 (World Anthropology Series)

BARABAS Alıcia La relocalizacion de poblaciones en el marco de las cien clas sociales America Indigena Mexico v 52 n 12 cnero/Jun 1992

BARABAS Alicia BARTOLOME Miguel Hydraulic development and etnocide the Mazatec and Chinantec people of Oaxaca Mexico Copenhagen IWGIA 1973 (Document 15)

BARABAS Alıcia BARTOLOME Miguel Antropologia y relocalızaciones Alteridades Iztapalapa UAM, v 2 n 4 p 5 I5 1992 (Reacomodos y constıuccion de presas)

BARABAS Alicia BARTOLOME Miguel Mediacion o autogestion grandes represas movimientos sociales y etnicidad Horizontes Antropologicos Porto Alegre v 3 n 6 p 38591997

BARTOLOME Leopoldo Aspectos sociales de la relocalizacion de poblacion afectada por la construccion de grandes represas In SUAREZ F, FRAN CO R COHEN, E (Org) Aspectos sociales de las grandes represas en Amern ca Latına Montevideo Fundacion de Cultura Universitana para CIDES (OEA) ILPES (ONU) 1984

BARTOLOME Leopoldo (Org) Relocalizados antropologia social de las po blaciones desplazadas Buenos Aures Ed del IDES 1985a (Coleccion Hombre y Sociedad 3)

BARTOLOME, Leopoldo Las relocalizaciones masivas como fenomeno so cial multıdimensional In (Org) Relocalizados antropologia so cal de las poblaciones desplazadas Buenos Aures Ed del IDES, 1985b (Coleccion Hombre y Sociedad 3)

BARTOLOME Leopoldo Estrategias adaptativas de los pobres urbanos el efecto entropico de la relocalızación compulsıva In (Org) $R e$ localzzados antropologia social de las poblaciones desplazadas Buenos Au res Ed del IDES 1985C (Coleccion Hombre y Sociedad 3)

BARTOLOME Leopoldo Grandes proyectos de desarrollo y desplazamıen tos poblacionales algunas claves para su comprension como procesos 
sociales complejos In CONGRESO ARGENTINO DE ANTROPOLOGIA SOCIAL 6 Mar del Plata 14 al 16 de septıembre 2000

BARTOLOME, Leopoldo Combatiendo a Leviatan la articulacion y difusion de los movimientos de oposicion a los proyectos de desarrollo hidroelectrico en Brasıl (1985 91) In BALAZOTE A CATULLO M RADOVICH $\mathrm{J}$ (Org) Antropologia y grandes proyectos en el Mercosur La Plata Minerva $200 \mathrm{I}$

BARTOLOME Miguel Presas y relocalızaciones de indigenas en America Latuna Alteridades Iztapalapa UAM v 2 n 4, p 1728 I992 (Reacomo dos y construccion de presas)

BARTOLOME Miguel BARABAS Alicia La presa Cerro de Oro y el ingentero el Gran Dios relocalizacion y etnocidıo Chinanteco en Mexico Mexico lnstituto Nacional Indigenista $1990 \vee 12$

BECKER Henk Social impact assessment method and experiences in Europe North America and the developing world London UCL Press 1977

BANCO INTERNACIONAL DE DESARROLLO (BID) Reasentamentos involun tanos en los proyectos del BID principios y lineamientos Washington DC BID 1996 Manuscrito

CATULLO Mana $\mathrm{R}$ Poder y participacion en proyectos de gran escala analisis comparativo de los procesos de relocalizacion por la construccion de la represa hidioelectıica binacional argentıno uruguaya de Salto Grande Tese (Doutorado em Ciências Sociars) - Instituto de Ciências Humanas Universıdade de Brasılıa, Brasılha 1996

CATULLO Marıa R Poder y niveles de decision en proyectos de gran escala In BALAZOTE A CATULLO M RADOVICH J (Org) Antropologia y grandes proyectos en el Mercosur La Plata Minerva 200I

CATULLO Maria R Ciudades relocalizadas una mirada desde la antropologia social Buenos Aures Biblos 2006

CATULLO Marıa R PATTI Beatrız Proceso de relocalızacion y nueva ciu dad Federacion Nueva Federacion, Entre Rios, Argentına In BALAZO TE A CATULLO M RADOVICH J (Org) Antropologia y grandes proyectos en el Mercosur La Plata Minerva 2001

CERNEA Michael Reasentamiento involuntario y desarrollo Finanzas y de sarrollo FMI Banco Mundial 1989a

CERNEA Michael Relocalizaciones involuntaras en proyectos de desarrollo linea mientos de politicas a ser aplicadas en proyectos financiados por el Banco Mundial Washington DC 1989 b (Documento Tecnico del Banco Mun dial 80S)

CERNEA Michael Social organization and development anthropology Swiss Institute for Development (SID) 1997

DE WET Cris Stress and environmental change in the analysis of commu nity relocation Human Organization v 47 n 2 Summer 1988

ESCUDERO C Involuntary resettlement in bank assisted projects an introduc tion to legal issues Washington DC The World Bank I988 
GIDDENS, Anthony The constitution of soctety Berkeley University of Califor nia Press 1984

GIDDENS, Anthony La vida en una sociedad post tradicional Revista de Oc cidente n 151 p $61-901993$

HELM Cecilia M Povos indigenas e projetos hidreletricos no Estado do Parana In FRERES STIPP N (Org) Analise ambrental usinas hidreletricas uma visão multıdısciplınar Londruna UEL NEMA 1999

HELM Cecilia $M$ Povos undigenas e projetos hidreletricos no Rio Tibagı In BALAZOTE A CATULLO M, RADOVICH J (Org) Antropologia y gran des proyectos en el Mercosur La Plata Munerva, 2001a

HELM Cecilia $M$ Kaungang e Guaranı da terra indigena Mangueurmha e a usına hıdreletrica Salto Santıago, no Rıo Iguaçu (PR) In REIS, M J BLOEMER N M S (Org) Hidreletricas e populações locats Florıanopolıs Cidade Futura Ed da UFSC, $2001 b$

HELM Cecilıa $M$ et al A implantação de usinas hidreletricas e os indigenas no Sul do Brasil Curitiba IAP GTZ 1998

JOYCE S Is it worth a dam? Environmental Health Perspectives v 105 n I0 p 10501055 Oct 1997

LUMSDEN Paul Towards a system model of stress feedback from an an thropological study of the impact of Ghana s Volta River Project In SARASON I SPIELBERG C (Ed) Stress and anxiety Washungton Heml sphere Publication Company 1975 v 2

PARTRIDGE Willam Reasentauniento de comunudades los roles de los gru pos corporativos en las relocalizaciones urbanas BARTOLOME Leopoldo (Org) Relocalizados antropologia social de las poblaciones desplazadas Buenos Aires Ed del IDES 1985 (Coleccion Hombre y Sociedad 3)

PLANT Roger Pobreza y desarrollo indigena algunas reflexiones Washington DC BID, 1998 Informe tecnico

RADOVICH Juan BALAZOTE Alejandro La etmicidad mapuche en un con texto de relocalizacion la represa de Piedra del Agula America Indigena Mexico v 5I n I enero/marzo 1991

REHNFELDT, Marilin Las tinieblas envuelven la tierra la construccion de la hidroelectrica Yacyreta y la relocalizacion de los indigenas Mbya Guaranı del Mabaepu In SANTOS S NACKE A (Org) Hidreletricas e povos ind genas Florıanopolıs Letras Contemporâneas 2003

REIS Marıa J Espaços vivências e identıdades os camponeses do Alto Uruguai e a hidreletrica de Ita In BALAZOTE A CATULLO $M$ RA DOVICH J (Org ) Antropologia y grandes proyectos en el Mercosur La Plata Minerva, 2001

REIS Mana J O reassentamento de pequenos produtores rurars o tempo da reconstrução e recriação dos espaços In REIS Marıa J, BLOEMER Neu sa Marıa Sens (Org) Hzdreletricas e populações locaıs Flonanopolıs Cidade Futura Ed da UFSC 2001 p 119166

RIBEIRO Gustavo L Proyectos de gran escala hacia un marco conceptual 
Aspectos teonco metodologicos sobre los procesos de reasentamiento poblacional e impactos sociales de la construccion de grandes represas hidroelectricas

para el analisis de una forma de produccion tempoiarıa In BARTOLO ME L (Org) Relocalizados antropologia social de las poblaciones despla zadas Buenos Aures Ed del IDES 1985 (Coleccion Hombre y Sociedad, 3)

RIBEIRO Gustavo L Da prefeitura ao Banco Mundial para uma metodolo gia de ação polıtıca com relação aos grandes projetos Brasılıa Fundação Universıdade de Brasílua 1990 (Serıe Antropologıca 97)

RIBEIRO Gustavo L Empresas transnacionais um grande projeto por dentro São Paulo Marco Zero ANPOCS 1991

ROBINSON Scott S El Proyecto Hidroelectrico Pangue Rio Bio Bio Chile y su importancia para el futuro de las obras de infraestructura Alterndades Iztapa lapa UAM v 2, n 4 p 8591 I992 (Reacomodos y construccion de presas)

SANTOS Silvio Coelho dos Construção de barragens e sociedades indigenas no Sul do Brasil In SEMINARIO EFECTOS SOCIALES DE LAS GRAN DES REPRESAS, 1983 Buenos Arres Anales Buenos Aures OEA CE PAL ILPES 1983 p 124

SANTOS Sílvio Coelho dos Presas y cuestiones socio ambientales en el Bra sll Alteridades Iztapalapa UAM v 2 n 4 p 3 I 371992 (Reacomodos y construccion de presas)

SANTOS Silvıo Coelho dos A implantação de usinas hidreletricas e os in dıos no Sul do Brasıl In HELM Cecilıa M (Coord) A implantação de usinas hidreletricas e os indios no Sul do Brastl Curitıba IAP GTZ 1996

SANTOS Silvio Coelho dos As hidreletricas os indios e o Direito In REU NIÃO BRASILEIRA DE ANTROPOLOGIA 20 Salvador BA 1418 de abril de 1996 Anats Salvador 1996

SANTOS Silvio Coelho dos Hidreletricas e suas consequências socioam bientais In VERDUM R (Org) Integração usinas hidreletricas e impactos socıoambientais Brasilıa INESC Fundação Heinrich Boll 2007

SANTOS Silno Coelho dos ASPELIN Paul El desarrollo hidroelectrico y los indigenas del Brasil Curculco Revista de la Escuela Nacional de Antropo logia e Historia Mexico v 4 n 131984

SANTOS Silvio Coelho dos HENRIQUES Karyn Hidreletricas e o processo de privatızação no cenario brasıleuro In BALAZOTE A CATULLO $M$ RADOVICH J (Org) Antropologia y grandes proyectos en el Mercosur La Pla ta Minerva 2001

SANTOS Silvio Coelho dos NACKE Aneliese A implantação das barragens na Bacıa do Rı Uruguat e suas implicações socıats a barragem de Machadınho e os undios de PI Ligeiro (RS) Florianopolis Ed da UFSC 1988 Relatorıo

SANTOS Silvio Coelho dos NACKE Aneliese (Org) Hidreletricas e povos in digenas Florianopolıs Letras Contemporâneas 2003a

SANTOS Silvio Coelho dos NACKE Aneliese A UHE Binacional Itaipu e os indios do Oco1 In lis Letras Contemporâneas 2003b

SANTOS Silvıo Coelho dos REIS Marıa Jose A construção de hıdreletrıcas 
um fenômeno social In REIS Mana Jose HELM Ceçlaa M (Coord) Hzdreletricas e reassentamento compulson to de populaçães aspectos sociocultu rais Curitiba IAP GTZ 1993

SCUDDER Thayer Resettlement In STANLEY F ALPERS $M$ (Ed) Man made lakes and human health New York Academic Press 1975

SCUDDER Thayer What it means to be dammed? the anthropology of large scale development projects in the tropics and subtropics Engineer ing and Science v 44 n 4 p 915 Apr 1981

SCUDDER Thayer, COLSON Elizabeth Long term field research in Gwem be Valley Zambia In FOSTER $G$ et al (Ed) Long term field research in social anthropology New York Academic Press 1978

SCUDDER Thayer COLSON Elizabeth From welfare to development a conceptual framework for the analysis of dislocated people In HANSEN A OLIVER SMITH A (Ed) Involuntary migration and resettlement Boul der Westview Press 1982 p 267287

SIGAUD Lygia Efettos socials de grandes projetos hidreletricos as barragens de Sobradınho e Machadınho Rıo de Janeiro Museu Nacıonal 1986 (Co municação 9)

SKLAR Leonard MCCULLY Patrich Damming the rivers The World Bank s lending of large dams International Rivers Network Working Paper Berkeley CA International Rivers Network n 5 Nov 1994

SUAREZ Francisco FRANCO Rolando COHEN Ernesto (Org) Efectos so ciales de las grandes represas en America Latma Buenos Aires CIDES ILPES 1984

WORLD BANK Involuntary resettlement the World Bank operational manual Washington DC The World Bank 2001 Bank Procedures (B P 4 12) \& (OP 4 12)

UQUILLAS Jorge DAVIS Shelton El Banco Mundial y los pueblos mdi genas de Amenca Latına In SUBCOMITE DE DERECHOS HUMANOS DEL PARLAMENTO EUROPEO Pueblos indigenas y organismos internaciona les 1996 Manuscrito

WERNER Dennis Psycho-social stress and the construction of a flood control dam in Santa Catarina Brazl Human Organization v $44 \mathrm{n} 2 \mathrm{p}$ 1611671985 